\title{
2.
}

\section{Nachtrag zu dem Eulerschen Lehrsatze von Polyëdern*).}

(Vom Herrn Prof. Dr. Hessel zu Marburg.)

\section{Der von Euler aufgestellte Lehrsatz **):} „, dafs bei jedem, von ebenen Flächen begrenzten Körper die Summe $\boldsymbol{E}+\boldsymbol{F}$ der Zahl $\boldsymbol{E}$ der Eeken und der Zahl $\boldsymbol{F}$ der Flächen um zwei gröfser als die Zahl $K$ der Kanten, also dafs $E+F=K+2$ sei, ist von Euler selbst anfangs ohne allgemein gültigen Beweis ausgesprochen ***), aber für viele einzelne Fälle bewiesen worden, und aus diesen einzelnen Beweisen wurde von Euler zuletzt der Schlufs gezogen, dals, weil in allen aufgeführten Fällen sich der Satz als wahr darstelle, kein Zweifel vorhanden sei über seine Richtigkeit für alle Ebenflächner $f$ )."

Andere ausgezeichnete Mathematiker (Legendre, Cauchy, Gergonne, Rothe und Steiner) $t_{t}$ ) haben Beweise für die allgemeine Gültigkeit des Satzes geliefert. Indessen leidet derselbe Ausnahmen.

*) Erst nach Absendung des Manuscripts za dieser Abhandlung, und nach langem vergeblichen Warten, erhielt der Verfasser von einer auswärtigen Bibliothek die Zeitschrift "Annales de mathématiques von Gergonne" und konnte in Band III. S. 169. sich überzeugen, dafs. bereits L'Huilier die Ausnahme gegen das fragliche Gesetz beleuchtet hat, wodurch das, was vorliegende Arbeit enthält, mit Ausnahme dessen, was die Andeutungen von Ausnahmen gegen das Eulersche Gesetz betrifft, die bedingt werden, wenn zwei oder mehirere Kanten eine gemeinschaftliche Kantenlinie, oder wenn zwei oder mehrere Ecken einen gemeinsamen Eck-Punct haben, den Werth der Neuheit verliert. Demungeachtet hält er es aber für nöthig, dafs auf jene Ausnahmen aufmerksam gemacht werde, da selbst neuere Schriftsteller sie nicht immer ausdrücklich berüicksichtigen.

**) Novi commentarii Acad. scient. imp. Petrop. tom. IV. pag. 119., wo or iu 5. 33. ausgedrückt ist auf folgende Weise:

"In omni solido hedris planis incluso aggregatum ex numero angulorum solidorum et ex numero hedrarum binario excedit numerum acierum."

****) Euler sagt a. a. O.: noin passe.

Fateri equidem cogor, me hujus theorematis demonstrationem firmam adhuc eruere

f) a. a. O. S.124.:

Cum igitur veritas propositionis in his omnibus casibus sibi constet, dubium est intlum, quin ea in omnibus omnino solidis locum habeat, sicque propositio sufficienter videtur demonstrata.

tt) Vergl. dieses Journal Band I. S. 228. und 364., wo auch die literarischen Hachweisungen zu finden sind. 
Euler führt in seiner Abhandlung bereits als Beispiel einen Körper an, welcher einspringende, rinnenartig vertiefte Kanten und einspringende Ecken hat, von welchen er die Gültigkeit seines Satzes darthut *), so dafs hieraus' sich ergeben mulste, die Beschaffenheit solcher Ecken und Kanten sei bei Aufstellung dieses Satzes berücksichtigt. Um aber die Ausnahmen ins Klare zu setzen, möge immer neben einer Gestalt, bei welcher das Eulersche Gesetz nicht gültig ist', eine ihr sonst ziemlich ähnliche aufgeführt werden, bei welcher es seine Gültigkeit hat.

Beispiele, wo $E+F=K+2$. Beispiele, wo $E+F=K+x$.

Fig. 2. (Taf. I.)

Ein vierseitig-säulenförmiger Körper mit vierseitiger, röhrenartiger Durchbohrung und ebenen vierseitig-ringfürmigen Endflïchen, hat 8 äufsere und 8 innere Ecken, 4 äufsere und 4 innere Seitenflïchen, 1 obere und 1 untere vierseitig-ringförmige EndHäche, 12 äufsere und 12 innere Kanten: bier ist also

$$
\begin{gathered}
E+F=B+2, \text { oder } \\
16+10=24+2 .
\end{gathered}
$$

\section{Fig. 4.}

Ein quaderähnlicherKörper, mit vierseitig - trichterartiger Durchbohrung, die nach unten zu vierseitig-säulenförmig wird, mit 8 äufseren und 12 inneren Ecken, 1 oberen und 1 unteren vierseitig ringförmigen Endflïche, 4 iufseren und 4 inneren verticalen Seitenflächen, 4 Trichter-Flächen, 20 horizontalen, 8 verticalen, 4 geneigten Kanten, so dafs also

$E+F=K+2$ oder $20+14=32+2$.

\section{Fig. 3.}

Ein vierseitig - säulenförmiger, an beiden Enden mit vierflächiger Facettirung versehener, vierseitig - röhrenartig-durchbohrter Körper hat 8 iiufsere und 8 innere Ecken, 4 äufsere und 4 innere Seitenflächen, 4 obere und 4 untere Flächen, 12 äufsere und 12 innere Kanten (wie in Fig. 2.) und noch 4 obere und 4 untere schiefe Kanten; so dafs

$$
\begin{aligned}
E+F & =\mathcal{R}+x, \text { hier also } \\
16+16 & =32+0 \text { ist. }
\end{aligned}
$$

Fig. 5.

Ein Körper, fast ähnlich dem Fig. 4., der aber, weil die Trichterflächen die obern Endflïchen ganz verdrängt haben, nur 8 äufsere und 8 innere Ecken, 1 untere vierseitige ringförmige Endfläche, 4 äufsere und 4 innere verticale Seitenfliichen, 4 Trichterliadhen, 16 horizontale, 8 verticale und 4 geneigte Kanten hat, so dafs $E+F=K+x$ oder $16+13=28+1$.

*) a. a. O. Fig. 6 . 
Fig. 6.

Ein Körper von der Form eines steinernen Wassertroges, dessen Mündung $a b c d$ mit 4 trapezförmigen Facetten umgeben ist. Er hat 8 iufsere Ecken wie go, 8 innere Ecken wie $a, 2$ horizontale, 8 verticale, 4 geneigte Fliichen, 16 horizontale, 8 verticale und 4 geneigte Kanten; folglich ist

$$
\begin{gathered}
E+F=K+2 \text { oder } \\
16+14=28+2 .
\end{gathered}
$$

Fig. 8.

Ein Körper von der Form einer aus drei Stufen bestehenden Treppe mit $3 \times 8=24$ Ecken, $3 \times 4+2=14$ Flächen und 36 Kanten, so dafs

$$
\begin{aligned}
F+F & =K+2 \\
24+14 & =36+2
\end{aligned}
$$

\section{Fig. 7 .}

Ein Körper von der Form eines gewöhmlichen steinernen Wassertroges mit ebener vierseitig-ringförmiger, oberer Horizontal-Fläche. Er hat 8 äufsere und 8 innere Ecken; 1 vierseitig - ringfürmige und 2 gewöhnliche horizontale Flächen; 8 verticale Flächen, 12 innere und 12 äufsere Kanten; mithin ist hier

$$
\begin{aligned}
E+F & =K+x \text { oder } \\
16+11 & =24+3
\end{aligned}
$$

\section{Fig. 9.}

Ein aus drei Stufen bestehender, ringsum freistehender - treppenartiger Körper *) mit $3 \times 8=24$. Ecken, $3 \times 5+1=16$ Flächen, $3 \times 12=36$ Kanten, wo also

$$
\begin{aligned}
E+F & =\boldsymbol{X}+\boldsymbol{x} \\
24+16 & =36+4
\end{aligned}
$$

Es mag dies hinreichen zu zeigen, dafs in der Gleichung

$$
E+F=K+x
$$

die Zahl $x$ nicht blofs nach Euler 2, sondern auch eine andere Zahl $0,1,2,3,4, \ldots$. u. s. w. sein kann. Es ist nun auch noch darzuthun, dals $x$ ne gativ sein könne.

Berücksichtigt man, dafs bei Fig. 5. der Werth $x=2-1=1$ und bei Fig. 3. der Werth $x=2-2=0$ ist, und beachtet man, dals z. B. die Fig. 3. als eine bestimmte Modification von Fig. 2. anzusehen ist, so wird einleuchten, dafs man durch ähnliche Modificationen aus Fig. 3. eino Gestalt erzeugen werde, wo $x=(2-2)-2=-2$ ist.

Ist daher $a b \in d i$ ofenmp $q g h i k$ (Fig. 10.) ein Körper ron der Art wie Fig. 3., der aber, statt ebener Flächen $f b c o$ und $m i h p$, vierseitige Facettirungen hat, die durch eine vierseitige säulenartige Durehboh-

*) Vergl. Hauy's Lehre ron der Entstehung des Zwölfrautenflächners aus dem Wüfel; treppenartige Kochsalztrichter u. s. w. 
rung $a \beta \delta \gamma \tau \varepsilon \rho \eta$ mit einander verbunden sind, so hat man 26 Flächen, 24 Ecken und 52 Kanten, so dafs hier die Gleichung

$$
\begin{aligned}
E+F & =K+x \text { sich in } \\
24+26 & =52-2
\end{aligned}
$$

verwandelt, und also $x=-2$ ist, wie es vorauszusehen war.

Dafs auch Gestalten sich bilden lassen, wo $x$ jede beliebige ganze Zahl mit dem Vorzeichen minus sein kann, braucht nun nicht erst noch weiter dargethan $\mathrm{zu}$ werden.

Bisher wurde nur von Gestalten gesprochen, deren Begrenzungsflächen unter sich in Zusammenhang stehen (gleichsam eine und dieselbe verschlungene Flächenkette bilden, deren Kanten als Verbindungs-Gelenke betrachtet werden können); in der Natur aber kommen auch Gestalten vor, wo dies nicht der Fall ist. Die Gestalt eines durchsichtigen Krystalls z. B., der einen minder durchsichtigen u. s. w. ringsum einschliefst, ist in seinem Innern, da wo er den eingeschlossenen berührt, gleichfalls mit Ebenen begrenzt (hat also gleichsam zwei Flächenketten, eine äufsere und eine innere), und ist deshälb als eigenthümliche Gestalt $\mathrm{zu}$ betrachten, die von der des nemlichen Krystalls, der den Einschlufs nicht enthält, stereometrisch wesentlich verschieden ist. Es wird daher nicht unzweckmälsig sein, ein möglichst einfaches bestimmtes Beispiel dieser Art näher zu beleuchten.

Ein durchsichtiger Flufsspathwirffel umschliefse ringsum einen undurchsichtigen Bleiglanzwürfel, so hat die Gestalt dieser Flufsspathmasse 8 äufsere und 8 innere Ecken, 6 äufsere und 6 innere Flächen, 12 äufsere und 12 innere Kanten.

Es ist also hier in

$$
\begin{aligned}
E+F & =K+x \\
16+12 & =24+4, \text { also } \\
x & =4
\end{aligned}
$$

mithin gleichfalls verschieden von 2 .

Sind mehrere einander nicht berührende Bleiglanzwürfel in einem und demselben Flufsspathwïrfel eingeschlossen, so wird, fǘr jeden Bleiglanzwürfel mehr, die Summe der Ecken and Flächen um $8+6=14$ vermehrt, die Summe der Kanten aber nur um 12, so dafs für jeden neuen Bleiglanzwürfel der Werth von $x$ um 14-12 oder. um zwei wächst, also stets eine gerade Zahl ist. 
Obgleich das bisher Vorgetragene genügend sein künnte, so mögen doch noch einige Worte folgen, tuber Fälle wo es zweifelhaft ist, ob man es mit einer oder mehreren Ecken, und mit einer oder mehreren Kanten zu thun habe; denn auch sie müssen in der Gestaltenlehre berücksichtigt werden. Es fragt sich nemlich: ist unter Ecke blofs der Punct zu verstehen, in welchem sich drei oder mehrere Kantenlinien eines Körpers schneiden, oder ist' die Ecke selbst zu verstehen, so, dafs der Fall denkbar ist, dafs zwei oder mehrere Ecken einen und denselben Scheitelpunct gemein haben. Nicht minder ist erst zu bestimmen, ob man unter Kante blofs die Kantenlinie verstehe, welche 2 Endpuncte verbindet, oder die Kante selbst, so, dafs der Fall gedacht werden kann, wo zwei oder mehrere Kanten eine und dieselbe Kantenlinie in derselben Längen-Erstreckung gemein haben.

Einen leichten anschaulichen Beweis des Satzes, dafs bei allen Ebenflächnern, in welchen jede gerade Linie, die zwei Ecken verbindet, ganz innerhalb des Körpers fällt, $E+F=K+2$ sei, und weitere Untersuchungen über die Bedingungen, unter welchen bei andern Ebenflächnern der Eul ersche Satz gilt oder nicht gilt, werde ich späterhin bekannt machen; hier möge in letzterer Beziehung nur ịn Allgemeinen Folgendes mitgetheilt werden.

Jeder Körper kann angesehen werden als eine stereometrische Summe (algebraische Summe, jedoch mit Rücksicht auf die Art der Verbindung oder Trennung) von zweien oder mehreren andern Körpera.

Für die Glieder $A, B$, einer solchen, nur aus 2 Gliedern bestehen. den stereometrischen Summe, d. h. für die beiden verbundenen Gestalten einzeln, seien folgende Gleichungen, die der Gleichung $E+F=K+x$ entsprechen, gültig:

$$
\begin{aligned}
& \text { 1. } e^{\prime}+f^{\prime}=k^{\prime}+a \text { für die Gestalt } A_{\text {g }}, \\
& \text { 2. } e^{\prime \prime}+f^{\prime \prime}=k^{\prime \prime}+b--\quad-B \text {, }
\end{aligned}
$$

und die entsprechende Gleichung für die Gesammtgestalt (für die stereometrische Summe) sei

8o ist aus 1. und 2.:

$$
\text { 3. } e+f=k+x \text {, }
$$

$$
\begin{aligned}
& \text { 4. }\left(e^{\prime}+e^{\prime \prime}\right)+\left(f^{\prime}+f^{\prime \prime}\right)=\left(k^{\prime}+k^{\prime \prime}\right)+(a+b) \text {, oder } \\
& \text { 5. }\left(e^{\prime}+e^{\prime \prime}\right)+\left(f^{\prime}+f^{\prime \prime}\right)-\left((a+b)=\left(k^{\prime}+k^{\prime \prime}\right)\right. \text {. }
\end{aligned}
$$

Cretle's Journal d. M. Bd. VIII. Beft. 1. 
Ist nun, wie in dem oben angeführten Beispiele des Flufsspathwürfels, der einen Bleiglanzwürfel umschliefst,

$$
\begin{aligned}
& \text { 6. } e=e^{\prime}+e^{\prime \prime}, \\
& \text { 7. } f=f^{\prime}+f^{\prime \prime}, \\
& \text { 8. } k=k^{\prime}+k^{\prime \prime} \text {. }
\end{aligned}
$$

so folgt aus (4., 6., 7. und 8.)

9. $x=a+b$,

so dafs, wenn $a=b=2$ ist, wie in jenem Beispiele, $x=2+2=4$ sein wirl.

Ist aber

also

10. $e^{\prime}+e^{\prime \prime}-e=\varepsilon$,

11. $f^{\prime}+f^{\prime \prime}-f=\varphi$,

12. $k^{\prime}+k^{\prime \prime}-k=\gamma$,

13. $e=e^{\prime}+e^{\prime \prime}-\varepsilon$,

14. $f=f^{\prime}+f^{\prime \prime}-\varphi$,

15. $k=k^{\prime}+k^{\prime \prime}-\dot{\gamma}$,

so folgt aus (5. und 15.)

$$
\text { 16. } k=\left(e^{\prime}+e^{\prime \prime}\right)+\left(f^{\prime}+f^{\prime \prime}\right)-(a+b+\gamma)
$$

und aus (13. und 14.)

$$
\text { 17. } e+f=\left(e^{\prime}+e^{\prime \prime}\right)+\left(f^{\prime}+f^{\prime \prime}\right)-(\varepsilon+\varphi) \text {. }
$$

Es folgt nun aus (16. und 17.)

mithin

18. $e+f-k=a+b+\gamma-(\varepsilon+\varphi)$,

$$
\text { 19. } e+f-k-x=a+b+\gamma-(a+\varphi)-x \text {. }
$$

Da nun

ist, so mufs auch

$$
e+f=k+x
$$

20. $z+\varphi=\gamma-x+(a+b)$ sein.

Sind mu die beiden Gestalten $A$ und $B$ solche, für welche $a=b=2$ ist, so hat man

also

$$
\text { 21. } \varepsilon+\varphi=\gamma-x+4 \text {, }
$$

$$
\text { 22. } x=\gamma-\varepsilon-\varphi+4 \text {. }
$$

Fïr Fig. 2. ist $\gamma=0, \varepsilon=0$ und $\varphi=2$; daher

$$
x=-2+4=2 \text {; }
$$

für Fig. 3. dagegen ist $\gamma=8, \varepsilon=8$ und $\varphi=4$, also hier

$$
x=8-8-4+4=0 \text {. }
$$

Für. Fig. 4. hat man $\gamma=0, \varepsilon=0, \varphi=2$, also wieder

$$
x=-2+4=2 \text {. }
$$


Fïr Fig. 5. aber ist auf ihuliche Weise

$$
\begin{aligned}
& \gamma=4, \varepsilon=4, \varphi=3, \text { also } \\
& x=4-4-3+4=1 .
\end{aligned}
$$

Neunt man einen Ebenflächner, für welchen das Eulersche Gesetz $(E+F=K+2)$ gilt, Eulerschen Ebenflächner (solidum Eulerianum), so kann man sagen:

Die Beantwortung der Frage, ob die stereometrische Summe zweier Eulerscher Ebenflïchner wieder einen Eulerschen Ebenflichner darstelte, hange von der Art und Weise ab, wie beide mit einander verbunden sind, nicht aber von ihrer sonstigen Beschaffenheit; und zwar stellt die stereometrische Summe zweier Eulerscher Ebenfläehner wieder einen Eulerschen Ebenflä chner dar, wenn ihre VerbindungsArt so ist, dafs die Summe der Zahl der durch die Verbindung verschwundenen *) Ecken und Flächen beider, gleich ist der um 2 vermehrten Zahl der verschwundenen Kanten; d. h. wenn

$$
\text { 23. } \varepsilon+\varphi=\gamma+2
$$

ist; denn aus (22.) folgt für $x=2$ auch

$$
\begin{gathered}
2=\gamma-\varepsilon-\varphi+4, \text { oder } \\
\varepsilon+\varphi=\gamma+2 .
\end{gathered}
$$

Wenn zwei Eulersche Ebenflächner von aufsen so aneinander gefügt werden, dafs eine $p$ seitige Begrenztungs - Fläche des einen zusammenfaillt mit einer ihr, als ebene Figur an sich betrachtet, gegenbildlich **) gleichen Begrenzungsflïche des andern, so dals diese beiden Begrenzungsflächen sich decken (mithin verschwinden), so/die Summe der beiden Gestalten, als ein neues Ganzes betrachtet, ein Eulerscher Ebenflächner.

Beweis 1. Wenn jede der $p$ Seiten der Vereinigungslliche eine Kante des neuen Körpers ist, so ist $\varphi=2, \varepsilon=\gamma=p$, und also

woraus das $\mathrm{zu}$ Beweisende folgt.

$$
\varepsilon+\varphi=\gamma+2 \text {. }
$$

*) Dies Verschwinden ron Ecken ist eine Verminderung der Summe der Ecken beider Körper. Die Vermehrung der Kanten - oder Eckensumme der Körper kommt natürlich als negative Verminderung in Rechnung.

**) Der Ausdruck gegenbildlich gleich (symmetrice aequalis), als dem obenbildlich gleich entgegengesetzt, ist in meiner Krystallometrio erläutert. Der ebené Spiegel lärst das Gegenbild des vor ihm stehenden Gegenstandes in sich wahrnehmen. 
2. Ist eine der $p$ Seiten jener. Vereinigungsfläche nicht eine Kante des neuen Körpers, so fällt auch eine der Flächen des einen der beiden verbundenen Körper so in die Verlängerung einer solchen des andern, daโs beide für 'den neuen ganzen Körper blofs für Eine Begrenzungsflïche zu zählen sind, und es ist dann $\varphi=3, \varepsilon=p, \gamma=p+1$, folglich $\varepsilon+\varphi=\gamma+2$.

3. Verschwinden zwei der Seiten der Vereinigungsfliiche, so dafs sie am Gesammtkörper nicht Kanten sind, so sind dieselben

a) entweder nicht aneinander stofsende Seiten; dann wiederholt sich blofs das vorhin (2.) Gesagte, und es ist $\varphi=4, \varepsilon=p, \gamma=p+2$, mithin $\varepsilon+\varphi=\gamma+2$.

b) oder sie stofsen aneinander; dann ist es möglich, dafs

a) entweder die Ecke, die jenem Vereinigungspuncte der verschwindenden Seiten angehört, nicht verschwindet, und dann ist der Fall blols ein solcher wie $2, a$.

B) oder diese Ecke verschwindet; dann fält aber noch eine, nicht in der Vereinigungs-Ebene liegende Kante des einen der beiden verbundenen Körper, in die Verlängerung einer solchen des andern. Es ist dann $\varphi=4, \varepsilon=p+1$ und $\gamma=p+3$, so dafs auch hier

$$
\varepsilon+\varphi=\gamma+2 \text {. }
$$

4. Verschwinden mehr, als zwei Seiten der Vereinigungs-Ebene, so wiederholt sich, für jede verschiedene solche Seite mehr, das unter (3.) Bemerkte, so dafs jedesmal $\varepsilon+\varphi=\gamma+2$ ist, weil jede Vermehrung, die auf der linken Seite dieser Gleichung um eine oder mehrere Einheiten statt hat, auch eine eben so grofse auf der rechten Seite, rücksichtlich auf den Werth von $\gamma$ bedingt.

5. Wenn keine Seite der Vreinigungs - Ebene als Kantenlinie auftritt und auch keiner der Scheitel der Winkel dieser Ebene als Eckpunot des neuen Körpers gezählt werden darf, so ist $\varphi=2+p, \varepsilon=2 p$ und $\gamma=3 p$, so, dafs auch hier $\varepsilon+\varphi=\gamma+2$ ist; wie sich schon aus (3. und 4.) ergiebt. 